\title{
Improved D2D Millimeter Wave Communications for 5G Networks Using Deep Learning
}

\author{
Ahmed Abdelreheem ${ }^{1,2}$, Ahmed S. A. Mubarak ${ }^{3}$, Osama A. Omer ${ }^{1}$, Hamada Esmaiel ${ }^{1}$, and Usama S. Mohamed ${ }^{4}$ \\ ${ }^{1}$ Dept. of Electrical Engineering, Faculty of Engineering, Aswan University, Aswan, Egypt. \\ ${ }^{2}$ Telecom Egypt \\ ${ }^{3}$ Dept. of Electrical Engineering, Faculty of Engineering, Sohag University, Sohag, Egypt. \\ ${ }^{4}$ Dept. of Electrical Engineering, Assiut University, Assiut, Egypt. \\ \{ahmed.abdelreheem, omer.osama, h.esmaiel\}@aswu.edu.eg, usama@aun.edu.eg
}

\begin{abstract}
Mode selection is normally used in conjunction with Device-to-Device (D2D) millimeter wave (mmWave) communications in 5G networks to overcome the low coverage area, poor reliability and vulnerable to path blocking of $\mathrm{mmWave}$ transmissions. Thus, producing a high-efficient D2D mmWave using mode selection based on select the optimal mode with low complexity turns to be a big challenge towards ubiquitous D2D mmWave communications. In this paper, low complexity and high-efficient mode selection in D2D mmWave communications based on deep learning is introduced utilizing the artificial intelligence. In which, deep learning is used to estimate the optimal mode $y$ in the case of blocking of mmWave transmission or low coverage area of $\mathbf{m m W a v e}$ communications. Then, the proposed deep learning model is based on training the model with almost use cases in offline phase to predict the optimal mode for data relaying high-reliability communication in online phase. In mode selection process, the potential D2D transmitter select the mode to transmit the data either based on dedicated D2D communication or through the cellular uplink using the base station (BS) as a relay based on several criteria. The proposed deep learning model is developed to overcome the challenges of selected the optimal mode with low complexity and high efficiency. The simulation analysis show that the proposed mode selection algorithms outperform the conventional techniques in D2D mmWave communication in the spectral efficiency, energy efficiency and coverage probability.
\end{abstract}

Keywords: D2D, mmWave, deep learning, mode selection, $5 G$ cellular networks.

\section{INTRODUCTION}

Fifth generation (5G) networks are demanded to support multi-Gbps data rates to address the incredible demand for tremendous user needs in the near future [1]. Immigration to millimeter wave (mmWave) band, 30 - $300 \mathrm{GHz}$ band be a promising candidate for enhancing the legacy cellular networks via support multi-Gbps data rates [2]. Recently, the integration between mmWave band and Device-to-Device (D2D) communications be strong candidate for enabling multi-Gbps data rates for $5 \mathrm{G}$ networks [3]. However, there big challenges in D2D mmWave communications such as weak link budget, high propagation loss and highly affected by rain and oxygen absorptions, so mmWave has doubts about the reliability in poor channel condition [4]. Analog beamforming (BT) technique based on steerable phased antenna array using a predefined codebook design with high antenna gains be enabler to overcome the poor channel condition in D2D mmWave communications [5]. The conventional BT using predefined codebooks at both transmitter (TX) and receiver (RX) which contains all beams with specific direction and beamwidth value using antenna weight vectors (AWVs). The BT is based on exhaustive search (EX) by scanning the whole $360^{\circ}$ around the TX/RX to obtain the best beam pair which maximizing the received power [3]. MmWave is the optimal candidate for D2D proximity services, and multi-Gbps data rates in D2D communications can be constructed. However, there are major challenges facing the construction of efficient mmWave D2D links. Blocking of communications between the devices is one of these main challenges due to the sensitivity of mmWave to blocking [5-7]. Another major challenges in construction of efficient mmWave D2D links is mode selection. Mode selection techniques is empowering the reliability of D2D mmWave communications. In the mode selection process, the potential D2D transmitter $\left(D_{t x}\right)$ select the mode to transmit the data either based on dedicated D2D communication or cellular uplink.

Actually, D2D pairs can select one of the following four modes of communication: 1) Pure cellular mode is used in case of high interference and low of available resources. 2) The partial cellular mode when D2D pairs can communicate through the base station (BS) without co-channel spectrum sharing. 3) Dedicated mode when the D2D pairs communicate with each other using dedicated spectrum resources. 4) Underlay mode in case of both D2D pairs and UEs share the uplink and downlink resources. However, mode selection proves challenging in mode selection processes such as resource management, network overloading and the complexity of the network management. There are many research efforts to overcome the challenges of mode selection in D2D mmWave communication while obtaining an acceptable complexity.

Motivated by the fact that mode selection in D2D communications need accurate decisions and predictions to can select the optimal mode that can guarantee improve the reliability of the system. We proposed a smart mode selection system based on deep learning, thanks to the deep learning feature as constructing intelligence model leads to accurate decisions and predictions will help in mode selection process. Deep learning is a part of artificial intelligence based on learning from the data referred to as training data, and able to 
independently adapt and classify patterns and make accurate decisions with the minimal human intervention [6].

\section{SYSTEM MODEL}

Assuming mmWave transmission between a D2D and the device to the base station (D2B) communications and the system model, including the $\mathrm{BT}$ of mmWave in $\mathrm{D} 2 \mathrm{D}$ and $\mathrm{D} 2 \mathrm{~B}$ connections. Considering the $\mathrm{BS}$ is equipped with $W_{B S}$ Antennas, and each device have $W_{D}$ antennas, and the coverage disk area $\mathcal{D}$ with a radius as $\mathcal{R}_{\mathcal{D}}$ and the $\mathrm{BS}$ is located at the origin of $\mathcal{D}$. Assuming that the devices are randomly deployed in the disc, following a homogeneous Poisson point process (HPPP). Consequently, the number of devices in $\mathcal{D}$ is Poisson distributed as follows $P(K$ devices in $\mathcal{D})=\frac{\mu^{K} e^{\mu}}{K !}$, where $\mu=\pi \mathcal{R}_{\mathcal{D}}{ }^{2} \lambda$. The BS serves UEs and several devices and each device in the system model can act as $D_{t x}$ or $D_{r x}$ and the potential D2D users $D_{t x}$ either establishes a mmWave communication through the BS directly known as cellular link or a direct D2D communication known as dedicated D2D communication based on the mode selection methodology.

\section{A. BT IN MMWAVE COMMUNICATIONS}

Considering the BT technique in D2D mmWave and D2B mmWave. Conventional BT using predefined antenna codebooks at TX/RX to obtain the optimal beam pairs for both $\mathrm{TX} / \mathrm{RX}$, the predefined codebook can be formulated as [8]:

$$
\mathcal{K}(w, \mathrm{~m})=j^{\text {floor }}\left\{\frac{\mathrm{w} \times \bmod \left(\mathrm{m}+\left(\frac{M}{2}\right), \mathrm{M}\right)}{\frac{M}{4}}\right\}
$$

where $w=0, \ldots, W-1, M=0, \ldots, M-1, j=\sqrt{-1}$, $\mathcal{K}(w, m)$ is the antenna weight corresponding to antenna element $w$ to accomplish BT steering in the $m$ direction, and $M$ and $W$ are the total numbers of steering beams and antenna elements respectively. The mmWave channel model consist of $L$ propagation paths between $\mathrm{BS}$ and $U E$ which can be formulated as follows [8]:

$$
\mathbf{H}=\frac{1}{\gamma} \sum_{\ell=1}^{L} \mu_{\ell} \boldsymbol{r}_{U E_{m}}\left(\phi_{\ell}\right) \boldsymbol{r}_{B S}^{H}\left(\theta_{\ell}\right)
$$

where $\gamma$ is the average path-loss, and ()$^{H}$ is the Hermitian transpose. The complex gain of path $\ell$ is given by $\mu_{\ell}$, and the array responses of $\mathrm{BS}$ and $D_{t x}$ are $\boldsymbol{r}_{B S}\left(\theta_{\ell}\right)$ and $\boldsymbol{r}_{U E_{m}}\left(\phi_{\ell}\right)$, respectively. The azimuth angles $\mathrm{AoD}$ and AoA are $\theta_{\ell} \in$ $[0,2 \pi]$ and $\phi_{\ell} \in[0,2 \pi]$, respectively, then [9]:

$$
\begin{aligned}
& \boldsymbol{r}_{B S}\left(\theta_{\ell}\right)=\left[1, e^{j \frac{2 \pi}{\lambda} e \sin \left(\theta_{\ell}\right)}, \ldots, e^{j\left(N_{B S}-1\right) \frac{2 \pi}{\lambda} e \sin \left(\theta_{\ell}\right)}\right]^{T} \\
& \boldsymbol{r}_{U E}\left(\phi_{\ell}\right)=\left[1, e^{j \frac{2 \pi}{\lambda} e \sin \left(\phi_{\ell}\right)}, \ldots, e^{j\left(N_{U E}-1\right) \frac{2 \pi}{\lambda} e \sin \left(\phi_{\ell}\right)}\right]^{T}
\end{aligned}
$$

Where the antenna elements spacing and the signal wavelength are given by $e$ and $\lambda$, respectively. Assuming 2-D BT, in case of the AWVs of both BS and $U E_{m}$ are directed in the $m_{t x}$ and $m_{r x}$ beam pattern, accordingly the $D_{t x} \mathrm{RX}$ symbol $y$ corresponding to the BS TX symbol $d$, can be stated as follows:

$$
y=\mathcal{K}_{r x}\left[:, m_{r x}\right]^{H} \mathbf{H} \mathcal{K}_{t x}\left[:, m_{t x}\right] d+\mathcal{K}_{r x}\left[:, m_{r x}\right]^{H} \boldsymbol{n},
$$

A. where $\boldsymbol{H}$ represents $W_{B S} \times W_{D}$ channel matrix. $\mathcal{K}_{t x}\left(\mathcal{K}_{r x}\right)$ is the TX $(\mathrm{RX})$ codebooks respectively, and $\mathcal{K}_{t x}\left[:, m_{t x}\right]\left(\mathcal{K}_{r x}\left[:, m_{r x}\right]\right)$ is the $\left[W_{B S} \times 1\right]\left(\left[W_{D} \times 1\right]\right) \mathrm{AWV}$ corresponding to $m_{t x}\left(m_{r x}\right)$ column in $\mathcal{K}_{\boldsymbol{t} \boldsymbol{x}}\left(\mathcal{K}_{\boldsymbol{r} x}\right)$, respectively. The noise vector is given by $\boldsymbol{n}$ with length $\left[W_{D} \times 1\right]$. We assume that the BS has perfect channel state information (CSI) of all the devices using methodology in [8].

B. Link Model in D2D MmWave Communications

Assuming $d$ is the distance between BS and $D_{r x}$, hence, the received power at $D_{r x}$ can be written in $\mathrm{dBm}$ as:

$$
\begin{aligned}
& P_{r}(d)=P_{t}+G_{T X}(\theta)+G_{R X}(\phi)-P L(d), \\
& P L(d)=P L_{o}\left(d_{o}\right)+10 n \log _{10}\left(\frac{d}{d_{o}}\right)+\chi_{\sigma},
\end{aligned}
$$

where $P_{t}$ and $P L(d)$ are the transmitter (TX) power and the potential path loss, respectively. $n$ and $\chi_{\sigma}$ indicate the path loss exponent and the log-norm shadowing term, i.e., $\chi_{\sigma} \sim$ $\mathcal{N}\left(0, \sigma_{\chi_{\sigma}}\right)$ where $\sigma_{\chi_{\sigma}}$ is the standard deviation of $\chi_{\sigma} . G_{T X}(\theta)$ and $G_{R X}(\phi)$ are the BT gain of the mmWave TX and RX, respectively, which can be expressed as [10]:

$$
\begin{gathered}
G_{T X}(\theta)=G_{o}-12\left(\frac{\theta-\varphi_{T X}}{\theta_{-3 d B}}\right)^{2}, \\
G_{o}=20 \log _{10}\left(\frac{1.6162}{\sin \left(\frac{\theta_{-3 d B}}{2}\right)}\right),
\end{gathered}
$$

Where $G_{o}$ is the maximum achievable gain of the antenna, $\theta_{-3 d B}$ and $\varphi_{T X}$ are half power beamwidth and the angle of the center of the beam, respectively [39]. Additionally, broadly, we can compute the signal-to-interference-plus-noiseratio (SINR) in mmWave communication between TX $i$ and receiver $(\mathrm{RX}) j$ as:

$$
\operatorname{SINR}_{i j}=\frac{P_{t_{i}}+G_{T X}(\theta)_{i j}+G_{R X}(\phi)_{i j}-P L(d)_{i j}}{\sum_{k \neq i} P_{t_{z}}+G_{T X}(\theta)_{z j}+G_{R X}(\phi)_{z j}-P L(d)_{z j}+n},(10)
$$

Where $z$ represents each interfering link in D2D and D2B mmWave communication.

\section{Blocking Model in MmWave Communications}

The distance is main parameter of mmWave blocking model, the blocking model is based on the distance between the transceivers and the shape of the blockers. The probability of LOS be blocked can be formulated as follows [10]:

$$
P(L O S)=e^{-\epsilon d}
$$

where $d$ denotes the distance between the transceivers, and $\epsilon$ is calculated based on the shape and the density of obstacles the buildings.

\section{PROBLEM FORMULATION}

In this section, we formulate the optimal RS in D2D mmWave communications based on the maximization problem for the throughput of the system. The $D_{t x}$ measure the link model with 
the target $D_{r x}$, and link model with $\mathrm{BS}$, and calculate the potential $S I N R$ for both $D_{r x}$ and BS. Finally, measure $P(L O S)$ between $D_{t x}$ and $D_{r x}$, and between BS and $D_{t x}$ and select the optimal mode selection for $D_{t x}$ as a direct connection with $D_{r x}$ or connect with $D_{r x}$ through the BS. All these metrics should be considered to jointly contribute in selecting the optimal mode that maximizes $\mathcal{R}$ which denotes the average system throughput.

$$
\mathcal{R}=\beta \log _{2}(1+\operatorname{SINR}),
$$

where $\beta$ is the bandwidth of the system, so the $\mathcal{R}$ can be formulated as:

$$
\mathcal{R}=\beta \log _{2}\left(1+\frac{P_{r}}{P_{\text {int }}+n}\right),
$$

Where the interference power between the devices can be rewritten as:

$$
P_{i n t}=\sum_{k \neq i} P_{t_{z}}+G_{T X}(\theta)_{z j}+G_{R X}(\phi)_{z j}-P L(d)_{z j}
$$

based on (5), the $\mathcal{R}$ can be expressed as:

$$
\mathcal{R}=\beta \log _{2}\left(1+\left|\frac{\mathcal{K}_{r x}\left[:, m_{r x}\right]^{H} \mathrm{H} \mathcal{K}_{t x}\left[:, m_{t x}\right]}{n}\right|^{2}\right),
$$

Consequently, the chosen mode guarantees the reliability of the system and increasing the total rate; finally, the optimal mode selection can be formulated as:

$$
M S^{*}{ }_{D_{t x}}=\underset{\forall(d, v, P(L O S), b)}{\arg \max }(\mathcal{R}),
$$

In this paper, we investigate the scheme that can guarantee determine the optimal mode selection in D2D mmWave communications with low complexity and high efficiency.

\section{PROPOSED DEEP LEARNING MODEL}

In this section, we give a detailed explanation of the structure of the deep learning model. Then, the detailed procedure of the proposed schemes for mode selection is addressed. For simplicity, in the following analytical analysis.

\section{A. Structure of Proposed Deep Learning Model}

The proposed deep learning scheme is constructed to find the optimal mode selection $M S^{*}{ }_{D_{t x}}$ that optimize the problem in (16). The proposed model is composed of several fully connected layers as shown in Fig. 1, each neuron in the layer is applied the activation function ReLU. In the following, a detailed explanation of proposed deep learning model. The data inputs are $\mathcal{Q}$ which is composed of $d, v, P(L O S)$ and $b$ the $S I N R$ is one of the main points in mode selection to determine the best mode, which reduce the interference which guarantee maximizing the $\mathcal{R}$ as given in (16) for each device is shaped as matrix with 5 columns as features and with rows $\mathcal{X}$ so, the input data $\boldsymbol{S}$ can be expressed as:

$$
\boldsymbol{S}=\left[\begin{array}{ccccc}
\operatorname{SINR}_{1} & d_{1} & v_{1} & P(L O S)_{1} & b_{1} \\
\vdots & \vdots & \vdots & \vdots & \vdots \\
\vdots & \vdots & \vdots & \vdots & \vdots \\
\operatorname{SINR}_{x} & d_{x} & v_{\chi} & P(L O S)_{\chi} & b_{\chi}
\end{array}\right],
$$

each element of the vector of the labeled data that indicate the projection of optimal $M S^{*}{ }_{D_{t x}}$ according to the features data $\boldsymbol{S}$. The actual best $M S^{*}{ }_{D_{t x}}$ according to actual measurements during the training process and a detailed explanation of training phase will be explained later. Consequently, the actual best $M S^{*}{ }_{D_{t x}}$ which guarantees (16) or labeled data can be expressed as:

$$
\boldsymbol{t}=\left[\begin{array}{c}
M S_{D_{t x 1}} \\
\vdots \\
\vdots \\
M S_{D_{t x} x}
\end{array}\right]
$$

Each neuron applied multiplication of the initialize weights with each input, then make a summation for each output process with the addition of biases and applied ReLU function to the output as shown in Fig. 1. Each hidden layer of full connected layers has a connection with the hidden nodes of the previous and next layers and fed into the ReLU layer, which provides non-linearity to extract meaningful features that are used to determine the optimal mode selection. Finally, the last layer of the deep learning model is determined the optimal $M S^{*}{ }_{t x}$.

\section{B. Training Phase of Proposed Deep learning scheme}

To use the proposed deep learning scheme, the deep learning model must first be trained, after which the trained model is used to determine the $M S^{*}{ }_{D_{t x}}$. As such, the training data are called features data of $d, v, P(L O S), b$ for several use cases of the devices under different channel condition, the distance between the devices, the mobility of the devices and the residual energy of the battery power of the devices in different cases are collected in the training and the corresponding best actual $M S_{D_{t x}}$ in each uses case. Consequently, the proposed model can learn the general best $M S_{D_{t x}}$ which maximizing the throughput of the system according to (16). During the training phase, we collected the training samples $\boldsymbol{S}$ and the label data $\boldsymbol{t}$. To avoid overfitting of the proposed deep learning model, we train only the appropriate number of channel samples and with sufficient to achieve accuracy performance as shown later in the simulation analysis. Additionally, the collected data will be

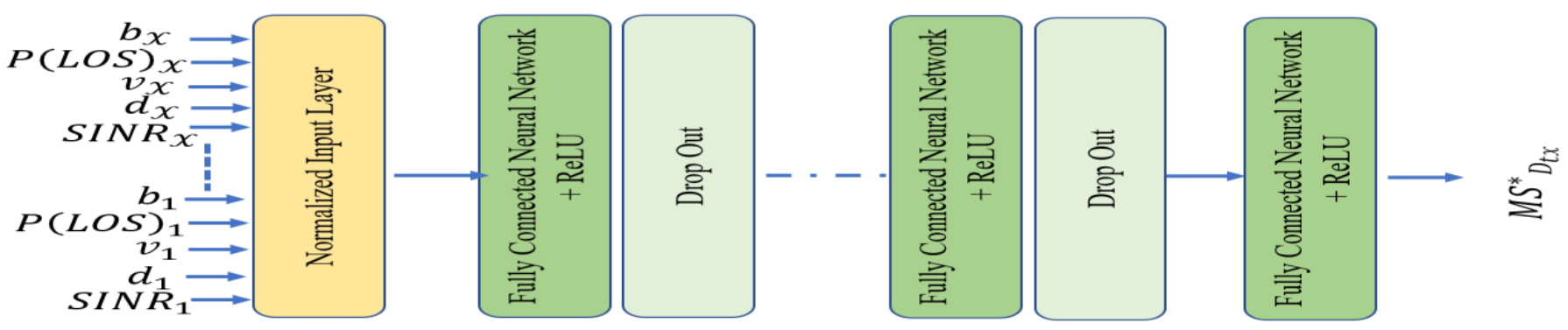

Fig. 1. Block diagram of the proposed deep learning model. 
normalized to ignore the effect of initializing the neural network weights and the outliers of the training samples. We Estimate the accuracy of the model that can be formulated as: $\mu=$ Mean squared error of $\left(M S_{D_{t x}}^{*}, M S_{D_{t x}}\right)$,

Algorithm: Proposed Mode Selection

Input: $\mathcal{X}$ : the number of training samples

Output: $M S^{*}{ }_{D_{t x}}$

The deep learning model start:

\section{A. Phase 1: Online learning:}

For $i=1: \mathcal{X}$

a. Input random values (training values): $d, v$ , $P(L O S), S I N R$ and $B$

b. Estimate $\mathcal{R}_{i}$ given in (15)

c. Determine $M S_{D_{t x_{i}}}$ in (16)

d. Estimated the accuracy as given in (19)

End

1. Construct the training data $\boldsymbol{S}$ as in (17)

2. Construct the labeled data $t$ as in (18)

\section{B. Online phase: Deep learning prediction}

Output: $M S^{*}{ }_{t x}$

End

V. SIMULATION ANALYSIS

In this section, we evaluate the performance of the proposed D2D mmWave communications scheme using numerical simulations compared to the standard IEEE 802.11ad [5] and in [11]. Deep learning model be implemented based on Python libraries and TensorFlow backend [12], [13]. For fair comparisons, we used the same simulation parameters given in [5] and [11] as follows: assuming simulation area equal to $5 \mathrm{~m}$ $\sim 100 \mathrm{~m}$ and the number of devices $N_{d}=10 \sim 200$ with equiprobable uniform distribution, both $60 \mathrm{GHz}$ and $5 \mathrm{GHz}$ carrier frequency are considered, Rayleigh fading channel is considered with $L=3$, AoAs/AoDs are assumed to be continuous in $[0,2 \pi]$ and assuming several cases of blockage Probability $P(L O S)=0.9,0.5$ and 0.1 . The transmit power $\left(P_{t}\right)=10 \mathrm{dBm}$ with Super-frame time $\left(T_{F}\right)=200 \mathrm{~ms}$. In the conducted simulations, we concern in measuring the throughput in bit/sec, the energy efficiency and the coverage probability of the compared D2D mmWave schemes. The throughput can be formulated as [10]: $\mathcal{T}=\frac{\mathcal{R}}{N_{d}}$, where $\mathcal{R}$ is the Spectral efficiency as in (15) and the total energy efficiency of the proposed scheme can be expressed as follows:

$$
\mathcal{E}=\frac{\mathcal{T}}{N_{d} \times P_{t} \times N_{f} \times T_{F}}
$$

The energy efficiency of the conventional mode selection can be formulated as follows:

$$
\mathcal{E}_{\text {conv }}=\frac{\mathcal{T}}{N_{d} \times P_{t} \times T_{F} \times P(L O S)+N_{d} \times P_{t} \times T_{F} \times(1-P(L O S))}
$$

The energy efficiency of mode selection can be formulated as follows:

$$
\varepsilon_{\text {proposed }}=\frac{\mathcal{T}}{P_{t} \times T_{F}},
$$

We first evaluate the accuracy of deep learning scheme according to (19) by investigated the required size $\boldsymbol{S}$ as in (18) to get satisfied performance of deep learning model. As shown in Fig. 6, as increasing the dataset or training samples, the accuracy of deep learning is increased until accuracy of the model is approximately saturated. Accordingly, we can reduce the complexity of training phase according to the saturated point where size $\boldsymbol{S}=30$ dataset of training data. In this section, we investigate the performance of the proposed mode selection scheme based on deep learning as shown in Figs.2, 3 and 4. In Figs. 2 and 3, we investigate the performance of the proposed mode selection scheme based on deep learning compared with schemes in [5] and [11]. The proposed mode selection scheme always shows a superior performance in the throughput and the energy efficiency compared with [5] and [11]. This come from, in [11] the mode selection scheme is based on the both distance between the devices and the distance to BS without investigate

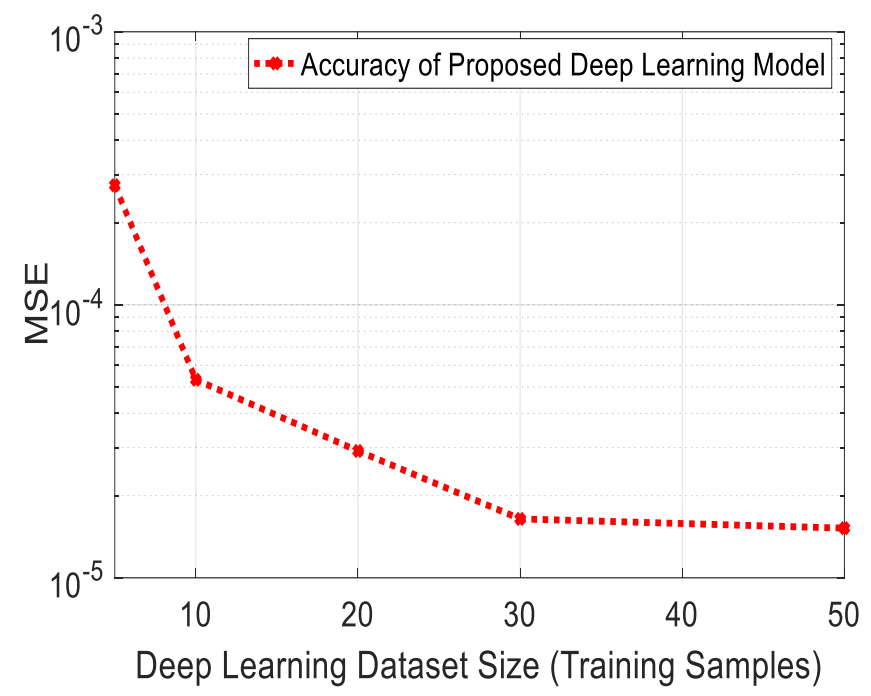

Fig. 2. The accuracy of proposed deep learning.

the channel condition of the device or the battery status of the device. Additionally, in [5] the conventional IEEE 802.11ad is based on D2D communications and only all possible communications come through the BS which act as relay for all devices, this methodology has no guarantee the link between the BS and devices is the best link in the case of the blocking of the direct path or high interference.

We study the coverage probability of the proposed scheme compared with schemes in [5] and [11]. The coverage probability can be defined as $P(\epsilon)=P(\mathcal{R} \geq \epsilon)$, and the outage happens if the rate of $D_{r x}$ falls below a certain threshold $\epsilon$. As shown in Fig. 4, The proposed mode selection scheme always shows a superior performance in the coverage probability compared with schemes in [5] and [11]. This come from, the proposed scheme based on smart system to take the decision on mode selection, select the optima mode which guarantee the optimal rate. In the other side, in schemes [5] and [11] is only on metrices that can't guarantee the best mode for obtain the high performance. 


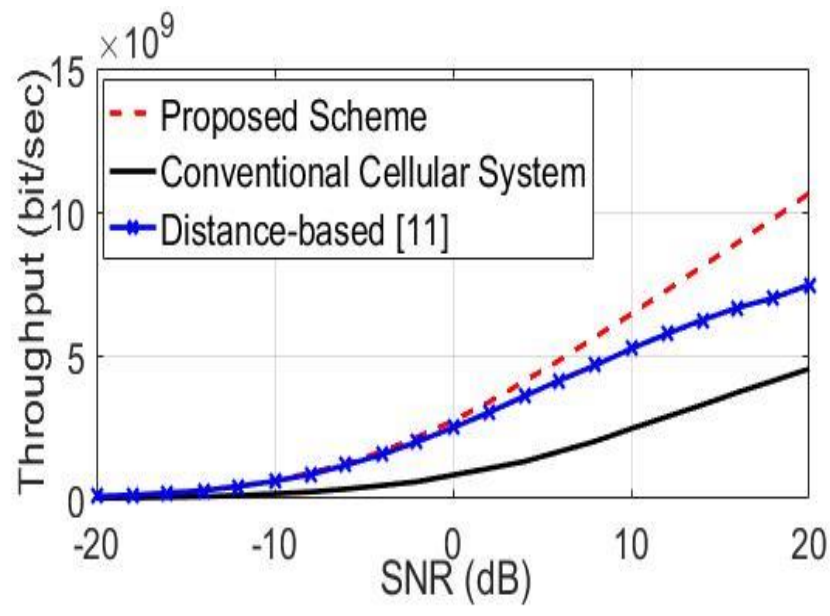

Fig. 3. Throughput efficiency comparisons in mode selection.

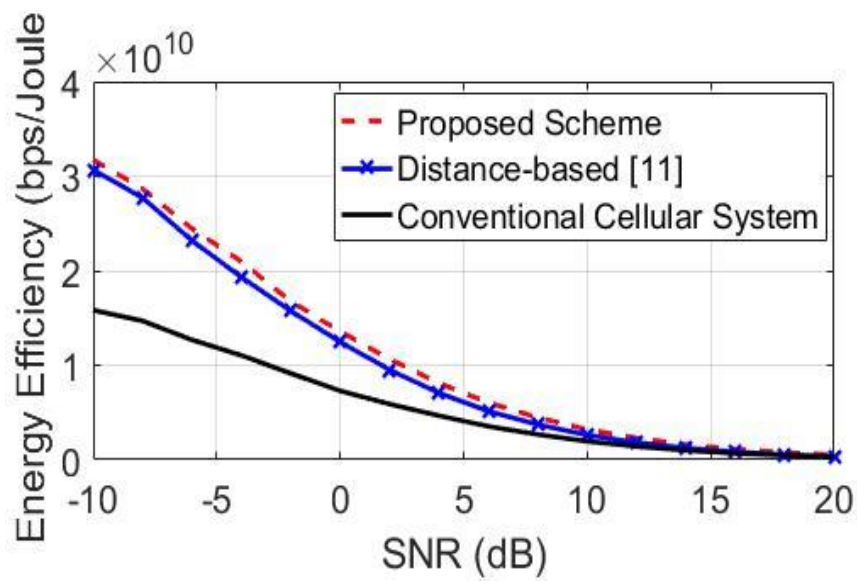

Fig. 4. Energy efficiency comparisons in mode selection.

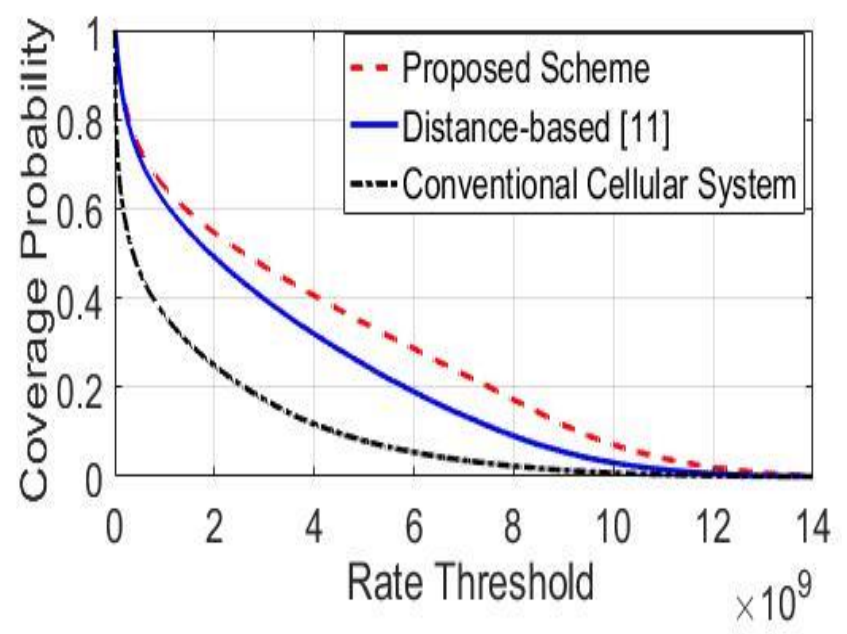

Fig. 5. Coverage probability in mode selection.

\section{CONCLUSION}

A smart mmWave communication scheme is proposed utilizing artificial intelligence. In which, the deep learning model is utilized to assist the D2D mmWave communication to overcome the challenges of selecting the optimal mode selection that guarantee the reliability of connectivity in D2D mmWave communication. Thus, we utilized the deep learning in mode selection methodology by developing mode selection process using deep learning to select the optimal mode to increase the high-reliability communication in D2D mmWave. We analyze the performance of the proposed scheme in the throughput, the energy efficiency and the coverage probability. The proposed D2D mmWave communication scheme obtains better performance than other schemes. Additionally, it can effectively withstand the effect of path blocking.

\section{REFERENCES}

[1] T. S. Rappaport, S. Sun, R. Mayzus, H. Zhao, Y. Azar, K. Wang, G. N. Wong, J. K. Schulz, M. Samimi, and F. Gutierrez, "Millimeter wave mobile communications for $5 \mathrm{G}$ cellular: It will work!," IEEE Access, vol. 1, pp. 335-349, 2013.

[2] R. Heath, N. Gonzalez-Prelcic, S. Rangan, W. Roh, and A Sayeed, "An Overview of Signal Processing Techniques for Millimeter Wave MIMO Systems," IEEE J. Sel. Top. Signal Process., vol. PP, no. 99, p. 1, 2016.

[3] S. Rangan, T. S. Rappaport and E. Erkip, "Millimeter-Wave Cellular Wireless Networks: Potentials and Challenges," in Proceedings of the IEEE, vol. 102, no. 3, pp. 366-385, March 2014.

[4] W. Roh, J. Y. Seol, J. Park, B. Lee, J. Lee, Y. Kim, J. Cho, K. Cheun, and F. Aryanfar, "Millimeter-wave beamforming as an enabling technology for $5 \mathrm{G}$ cellular communications: Theoretical feasibility and prototype results," IEEE Commun. Mag., vol. 52, no. 2, pp. 106-113, 2014.

[5] IEEE 802.11ad Part 11: "Wireless LAN medium access control $(M A C)$ and physical layer (PHY) specifications amendment 3: enhancements for very high throughput in the $60 \mathrm{GHz}$ band," 2012.

[6] W. G. Hatcher and W. Yu, "A Survey of Deep Learning: Platforms, Applications and Emerging Research Trends," in IEEE Access, vol. 6, pp. 24411-24432, 2018.

[7] X. Lin, J. G. Andrews and A. Ghosh, "Spectrum Sharing for Device-to-Device Communication in Cellular Networks," in IEEE Transactions on Wireless Communications, vol. 13, no. 12, pp. 6727-6740, Dec. 2014.

[8] A. Abdelreheem, E. M. Mohamed and H. Esmaiel, "Millimeter wave location-based beamforming using compressive sensing," 2016 28th International Conference on Microelectronics (ICM), Giza, 2016, pp. 213-216.

[9] A. Abdelreheem, E. M. Mohamed and H. Esmaiel, "LocationBased Millimeter Wave Multi-Level Beamforming Using Compressive Sensing," in IEEE Communications Letters, vol. 22, no. 1, pp. 185-188, Jan. 2018.

[10] Abdelreheem, Ahmed; Mohamed, Ehab Mahmoud; Esmaiel, Hamada. "Adaptive location-based millimetre wave beamforming using compressive sensing based channel estimation," IET Communications, 2019, vol. 13, issue 9, p. 12871296.

[11] H. Ding, S. Ma, and C. Xing, "Feasible D2D communication distance in D2D-enabled cellular networks," in IEEE International Conference on Communication Systems (ICCS), Macau, Nov. 2014.

[12] A. Abdelreheem, O. A. Omer, H. Esmaiel and U. S. Mohamed, "Deep Learning-Based Relay Selection In D2D Millimeter Wave Communications," 2019 International Conference on Computer and Information Sciences (ICCIS), Sakaka, Saudi Arabia, 2019, pp. 1-5.

[13] TensorFlow: open-source machine learning library, [Online]. Available: https://www.tensorflow.org/tutorials/ , Last access (2019, April 5). 